\title{
L'IMPORTANCE DU SUIVI PSYCHOLOGIQUE ET NUTRITIONNEL APRÈS CHIRURGIE BARIATRIQUE : REVUE DE LA LITTÉRATURE
}

\section{ARTICLE ORIGINAL}

DENDASCK, Carla Viana ${ }^{1}$, SANTOS, Rogério Bongestab dos², SANTOS, Vitor Maia ${ }^{3}$, ANDRADE, Tadeu Uggere de ${ }^{4}$, PFLUG, Adriano Ribeiro Meyer ${ }^{5}$

DENDASCK, Carla Viana. Et al. L'importance du suivi psychologique et nutritionnel après chirurgie bariatrique : revue de la littérature. Revista Científica Multidisciplinar Núcleo do Conhecimento. Année. 06, Ed. 10, vol. 01, p. 20 à 44. Octobre 2021. ISSN: 2448-0959, Lien d'accès: https://www.nucleodoconhecimento.com.br/psychologie-fr/suivi-psychologique, DOI: 10.32749/nucleodoconhecimento.com.br/psychologie-fr/suivi-psychologique

\footnotetext{
${ }^{1}$ Théologien, docteur en psychanalyse clinique. Il travaille depuis 15 ans avec la Méthodologie Scientifique (Méthode de Recherche) dans I'Orientation de Production Scientifique pour les étudiants en Master et Doctorat. Spécialiste en études de marché et recherche en santé, doctorant en communication et sémiotique (PUC SP).

${ }^{2}$ Diplômé en médecine de l'École des sciences de Santa Casa de Misericórdia de Vitória - EMESCAM. Résidence médicale en chirurgie générale à l'Hôpital Santa Casa de Misericórdia de Vitória (HSCMV). Post-graduat en Thérapie Nutritionnelle à l'EMESCAM. Diplômé en Nutrologie de la Faculté des Sciences Médicales de Santa Casa de Misericórdia de São Paulo (FCMSCM-SP/ABRAN). Spécialiste en Nutrologie par l'Association Brésilienne de Nutrologie (ABRAN). Spécialiste en thérapie nutritionnelle par la Société brésilienne de nutrition parentérale et entérale (SBNPE/BASPEN).

${ }^{3}$ Psychiatre. Spécialiste en psychiatrie et psychiatrie légale par l'Association brésilienne de psychiatrie (ABP). Docteur au Ministère de l'Economie (ME). Étudiant diplômé en Master en Sciences Pharmaceutiques à I'Université de Vila Velha (UVV). Secrétaire-directeur adjoint de l'Association psychiatrique Espírito Santo (APES). Secrétaire de la Commission de psychiatrie du travail de l'ABP. Professeur au cours de troisième cycle en psychiatrie à l'Université de Vila Velha (UVV). Diplôme d'études supérieures en médecine du travail et diplôme d'études supérieures en expertise médicale.

${ }^{4}$ Doctorat en Sciences Physiologiques - Université Fédérale d'Espírito Santo, UFES. Master en Management International - Steinbeis University Berlin, STW-SIBE. Master en Sciences Physiologiques - Université Fédérale d'Espírito Santo, UFES. Spécialisation en Gastronomie Fonctionnelle - Collège Méthode de São Paulo, FAMESP. Diplôme en Pharmacie - Faculté de Pharmacie et Biochimie d'Espírito Santo, FAFABES.

${ }^{5}$ Docteur par FMUSP. Diplômé en médecine de la Faculté de médecine de l'Université de São Paulo (FMUSP). Résidence médicale en chirurgie générale et chirurgie générale avancée à l'Hôpital das Clinicas, Faculté de médecine, Université de São Paulo (HC-FMUSP). Chirurgien général par le Collège brésilien des chirurgiens $(C B C)$, Chirurgien du système digestif par le Collège brésilien de chirurgie digestive (CBCD). Chirurgie bariatrique (SBCBM) Médecin assistant de la clinique chirurgicale Division III de I'Institut central de l'Hôpital das Clínicas de la FMUSP.
}

RC: 98481

Lien d'accès: https://www.nucleodoconhecimento.com.br/psychologie-fr/suivi-psychologique 


\section{RÉSUMÉ}

L'obésité est une maladie chronique multifactorielle. Le patient peut présenter plusieurs conditions cliniques qui lui sont associées. Le tableau clinique est complexe, ce qui peut entraver l'utilisation de traitements et de méthodes conventionnels. La chirurgie bariatrique est un traitement efficace à long terme pour cette condition. Basé sur une revue de la littérature, l'article vise à discuter de l'importance du suivi psychologique et nutritionnel post-bariatrique. La question fondamentale qui sous-tère cette étude est la suivante: comment les stratégies typiques de la période postopératoire peuvent-elles contribuer à ce que l'individu subisse une intervention chirurgicale accorde une plus grande importance à son alimentation et à sa santé mentale? La stratégie de traitement la plus efficace a été la chirurgie bariatrique, cependant, l'évaluation multidisciplinaire qui note l'état biologique, social et psychologique du patient est fondamentale, car les changements psychopathologiques sont communs aux personnes obèses. Bien que la chirurgie bariatrique contribue à l'amélioration de la qualité de vie des patients, il faut accorder une plus grande attention au traitement postopératoire. Ces personnes doivent être encouragées à poursuivre leur suivi nutritionnel et psychologique pendant la période postopératoire, afin qu'il soit possible de prévenir d'éventuelles complications à moyen et à long terme.

Mots-clés : Obésité, Santé mentale, Surveillance nutritionnelle, Bariatrie.

\section{INTRODUCTION}

L'obésité est une épidémie croissante, et facilite ainsi le développement de comorbidités, telles que le diabète et l'hypertension artérielle systémique (RODRIGUES et al., 2017). Cela atteint l'échelle mondiale, environ deux milliards de personnes sont obèses ou en surpoids. En 2008, on estimait qu'environ $43 \%$ de la population adulte était obèse, mais ce nombre a augmenté, atteignant 54 \% en 2015 (RODRIGUES et al., 2017). Au Brésil, il a été identifié que, selon les données du Système de surveillance des facteurs de risque et de protection des maladies chroniques par enquête téléphonique, $53 \%$ de la population adulte était considérée RC: 98481 
comme en surpoids et $18,9 \%$ obèse (BRASIL, 2017). Avec l'augmentation de l'obésité, le nombre de chirurgies bariatriques a donc augmenté, comme l'a souligné la Société brésilienne de chirurgie bariatrique et métabolique (SBCBM, 2017). Le Brésil est le deuxième pays au monde en nombre de chirurgies bariatriques pratiquées chaque année. Comparativement à 2015, il y a eu une augmentation de $7,5 \%$ des chirurgies pratiquées (SBCBM, 2017). La perte de poids après la chirurgie reflète une amélioration significative des comorbidités, telles que le diabète, la dyslipidémie, I'hypertension et l'apnée du sommeil (BUCHWALD et al., 2004). Cependant, il convient de souligner que le traitement chirurgical n'est qu'une partie liée au traitement intégral de l'obésité et que le suivi nutritionnel et psychologique après la chirurgie est d'une importance fondamentale. Par conséquent, il faut investir dans des stratégies visant à promouvoir la santé et les soins cliniques longitudinaux (BRASIL, 2017). Le patient qui vise à effectuer l'opération doit répondre à certaines exigences, quelle que soit la technique chirurgicale, telles que l'IMC supérieur à $40 \mathrm{~kg} / \mathrm{m}^{2}$ ou l'IMC supérieur à 35 $\mathrm{kg} / \mathrm{m}^{2}$ en présence de comorbidités liées au surpoids (SBCBM, 2006). La liste des comorbidités a été élargie et une plus grande partie de la population a été envisagée (BRASIL, 2017).

Chaque patient subissant ce type de chirurgie doit faire l'objet d'un suivi multidisciplinaire. L'objectif est l'évaluation, l'orientation et le zèle pour la relation équipe-patient, car c'est un moyen de promouvoir l'efficacité du traitement dans la période postopératoire (GORDON; KAIO; SALLET, 2011). Le nutritionniste joue un rôle essentiel dans les périodes pré et postopératoires. Le conseil nutritionnel dans la période préopératoire est intrinsèquement lié à l'augmentation du potentiel de succès dans la période postopératoire (CRUZ, 2004). Une étude liée à la récidive du poids a conclu que $39 \%$ des patients opérés avaient pris du poids (surtout après 12 mois de chirurgie). Parmi les facteurs qui ont soulevé le problème, il y a la faible participation aux consultations nutritionnelles pendant la période préopératoire, qui a abouti à une récidive du poids (BARDAL; CECATTO; MEZZOMO, 2016). Les personnes qui passent par un processus d'éducation nutritionnelle et de conseil ont de meilleurs résultats. 
Les conseils psychologiques et nutritionnels devraient être avant et après la chirurgie. La régurgitation qui affecte habituellement les patients qui n'ont pas la bonne habitude de mâcher est courante chez les patients qui ne subissent aucun type de suivi multidisciplinaire (COOPER et al., 1999). Cependant, il y a un abandon élevé dans le suivi nutritionnel postopératoire, ce qui peut entraîner des complications métaboliques et une récidive de poids (MENEGOTTO et al., 2013). Pour ces raisons, l'ajustement de l'alimentation dans la période préopératoire est d'une importance fondamentale, car, par conséquent, les nouvelles habitudes alimentaires posées par les exigences de la période postopératoire seront plus propices à la mise en pratique (RODRIGUES et al., 2017). Des ajustements à vie qui incluent le comportement alimentaire et les activités physiques sont nécessaires pour une perte de poids réussie et pour prévenir les complications à long terme (COSTA, 2013).

Dans l'évaluation nutritionnelle préopératoire, en plus de l'anamnèse nutritionnelle complète, l'apport alimentaire, les tests de laboratoire, le diagnostic des comorbidités et les antécédents de poids sont effectués. II est apprécié pour la correction des carences nutritionnelles fréquemment identifiées. Les patients obèses subissent parfois plusieurs régimes restrictifs et peuvent donc être considérés comme des personnes souffrant de malnutrition (DOLNE; SOUZA, MATTE, 2020). Cependant, d'autres questions relatives à la chirurgie devraient être évaluées, telles que la disponibilité pour le changement et l'évaluation des connaissances générales sur la nutrition et la chirurgie elle-même (AILLS et al., 2008). Ces informations permettent au professionnel d'identifier les obstacles possibles qui peuvent interférer avec la perte de poids ou entraîner des conditions postopératoires négatives, telles que l'abus d'alcool et d'autres drogues. En outre, le patient doit être préparé aux premières phases de l'évolution du régime postopératoire (COSTA, 2013). Ainsi, l'éducation nutritionnelle préopératoire devrait favoriser l'amélioration des résultats chirurgicaux.

Le soutien psychologique fourni au patient qui est candidat à la chirurgie bariatrique (pré- et postopératoire) a des objectifs spécifiques (ROCHA; COSTA, 2012). Parmi eux, nous soulignons les connaissances sur les habitudes alimentaires, les modes de vie, la relation avec la nourriture et les émotions impliquées dans ces interactions 
(KORTCHMAR et al., 2018). Après cette connaissance, le psychologue devient capable de connaître et de tracer une ligne de continuité à partir du suivi psychologique postopératoire, car les chances de succès avec un traitement chirurgical sont plus élevées (SILVA, 2015). Connaissant les facteurs qui peuvent générer des difficultés dans ce processus d'adaptation, le patient bénéficie d'une plus grande sécurité et d'une attention accrue dans ces aspects qui le placeraient dans la plage de risque de prise de poids (FAGUNDES; CAREGNATO; SILVEIRA, 2016).

Le soutien psychologique est donc un moyen de connaître les facteurs qui empêchent l'efficacité du traitement post-bariatrique (MENSORIO, 2013). Compte tenu de ce scénario, l'expérience clinique a montré que le succès du traitement chirurgical de l'obésité ne dépend pas seulement de la qualité technique de l'intervention chirurgicale. Il s'agit donc d'un processus qui dépend de la capacité du patient à s'adapter à un nouveau régime alimentaire, à un nouveau corps et à un nouveau mode de vie (MAGDALENO JR; CHAIM, TURATO, 2009). Les difficultés à changer radicalement la qualité, ainsi que la quantité de ce qui est ingéré, peuvent amener les personnes qui ont subi une intervention chirurgicale à ne pas perdre de poids ou, sinon la totalité, du poids perdu (KORTCHMAR et al., 2018). Ce processus peut générer un sentiment d'échec et de désespoir, pour cette raison, le soutien psychologique est essentiel.

Le maintien du poids à long terme est essentiel (KULICK; HARK; DEEN, 2010). Un autre point à évaluer est la perte de poids préopératoire. II n'y a pas suffisamment de preuves pour soutenir cette perte de poids avant la chirurgie, car lorsqu'elle est effectuée, elle favorise un retard inutile pour le traitement du patient (KIM et al., 2016). Des études affirment qu'un régime hypocalorique préopératoire peut causer certaines carences nutritionnelles préexistantes, ainsi que la perte de masse musculaire peut conduire le patient au risque de malnutrition, cependant, d'autres études comprennent que la réduction du volume abdominal et hépatique a de meilleurs résultats à long terme (EDHOLM et al., 2011; THIBAULT et al., 2016). Compte tenu du présent article, le présent article pose une question fondamentale: comment les stratégies typiques de la période postopératoire favorisent-elles une plus grande prise en charge de 
l'alimentation et de la santé mentale chez les patients? Pour y répondre, une revue de la littérature a été réalisée, dans le but de discuter de l'importance du suivi psychologique et nutritionnel post-bariatrique.

\section{L’OBÉSITÉ ET SES IMPLICATIONS}

L'obésité compromet considérablement la qualité de vie et favorise la prédisposition à d'autres maladies non transmissibles, en particulier celles qui nuisent à la santé mentale de l'individu (MARTINS; MIYAZAKI, 2019). Pour cette raison, l'obésité devrait être considérée comme un problème de santé publique, étant donné que la vie quotidienne des gens est compromise.

Afin de mieux délimiter le contexte de cette étude, les implications de la chirurgie bariatrique, l'équipe multidisciplinaire qui fait partie de cet univers et les soins prodigués par le psychologue et le nutritionniste après la chirurgie, il est nécessaire de comprendre la multifactorialité qu'implique l'obésité et, par conséquent, le patient bariatrique (GORDON; KAIO; SALLET, 2011). Par conséquent, au départ, c'est un patient qui a besoin d'un suivi psychologique pour être effectué en continu, car la réalisation de ce type de chirurgie nécessite un changement d'habitude non seulement du patient, mais également du groupe social dont il fait partie (KORTCHMAR et al., 2018).

II est à noter que les questions familiales et interpersonnelles, les aspects pertinents de la santé mentale, tels que l'estime de soi, l'équilibre affectif et la perception de la réalité, et enfin le suivi par une équipe multidisciplinaire sont quelques-uns des termes qui commencent à intégrer la vie de la personne obèse, avant ou après le processus chirurgical (MARTINS; MIYAZAKI, 2019). Ainsi, il est nécessaire de comprendre, dans ce scénario, la fonction sociale assumée par le psychologue et le nutritionniste et à quel point sa performance est essentielle.

La prévalence accrue du surpoids et de l'obésité dans le monde a inquiété les agences de santé publique, ce qui a entraîné une augmentation des dépenses publiques. Cependant, il existe une justification plausible: des efforts et une volonté de fonds sont RC: 98481 
nécessaires dans de nombreux projets de recherche traitant du thème de l'obésité, car elle augmente considérablement chaque année (RASOULI et al., 2007). Les chiffres ne sont pas typiques de la réalité brésilienne, car, en raison de certaines habitudes et habitudes alimentaires, les chiffres sont élevés dans le monde entier.

Des pays comme le Brésil, le Canada et la France traitent avec un pourcentage élevé de personnes en surpoids (environ 40\%). D'autres pays, comme les États-Unis et l'Allemagne, plus de $65 \%$ de la population est classée de cette manière. Compte tenu de ce scénario, il est entendu que cette réalité sociale se reflète dans le scénario politico-économique, car une surcharge est créée dans les systèmes de santé de manière plus significative, c'est-à-dire qu'elle est investie dans les soins préventifs, les consultations médicales, les médicaments, les hospitalisations, les examens, les chirurgies, entre autres possibilités (MARTINS; MIYAZAKI, 2019). D'autres problèmes indirects contribuent à cette augmentation, tels que l'absence de travail, la perte de temps libre, les morbidités et la mortalité (MELO, 2011). L'étude de Kac; Sichieri e Gigante (2007) a identifié qu'il est moins coûteux de traiter l'obésité que de traiter les comorbidités qui en sont à l'origine. Cependant, des mesures préventives plus efficaces et plus actuelles sont nécessaires avec le traitement.

Ces mesures préventives, afin d'être plus efficaces, devraient promouvoir certains types de pratiques. Parmi eux, il est nécessaire de favoriser le dialogue entre les multiples pratiques complémentaires entre elles, en particulier celles qui impliquent conjointement les travaux du psychologue et du nutritionniste (GORDON; KAIO; SALLET, 2011). L'obésité doit être insérée dans un contexte dynamique dans lequel la maladie existe, persiste et crée des mécanismes qui partent d'une mauvaise alimentation, d'une faible dépense énergétique, d'un mode de vie sédentaire et d'un apport calorique élevé (MARTINS; MIYAZAKI, 2019). C'est un médium qui doit également être traité et, par conséquent, la population doit être interprétée dans une perspective intégrale, considérant qu'ils sont insérés dans une culture de consommation qui doit être réorganisée. Par conséquent, les habitudes qui assurent le bien-être collectif doivent être stimulées. Dans ce contexte, la chirurgie bariatrique émerge et agit comme une alternative plausible.

RC: 98481

Lien d'accès: https://www.nucleodoconhecimento.com.br/psychologie-fr/suivi-psychologique 
Cependant, le bariatrique en lui-même n'est pas en mesure de résoudre ce problème de santé publique qui affecte la qualité de vie d'un certain nombre de personnes, non seulement d'un point de vue local, mais à l'échelle mondiale. Par conséquent, le suivi psychologique et nutritionnel est d'une importance primordiale et, par conséquent, il faut investir dans un traitement de santé basé sur une approche multidisciplinaire (GONÇALVES; KOHLSDORF; PEREZ-NEBRA, 2020). La surveillance étroite des professionnels de la santé de plusieurs domaines et de la famille elle-même est essentielle pour que le traitement auquel le patient a été soumis soit efficace à long terme. La chirurgie bariatrique ne se limite pas à la perte de poids (FANDIÑO et al., 2004). II vise avant tout l'amélioration de l'état biopsychosocial de l'individu, étant une question qui imprègne la qualité de vie (COSTA et al., 2009). Considérant que le succès de la chirurgie bariatrique dépend, en plus de l'équipe multidisciplinaire, de la motivation du patient, ce qui implique un changement dans les modes de vie et de consommation (CAMPOS et al., 2016).

\section{LES CARACTÉRISTIQUES DE LA CHIRURGIE BARIATRIQUE}

L'obésité atteint des sommets élevés à l'échelle mondiale, ce qui a attiré l'attention des autorités et nécessite de multiples efforts de la part de professionnels et d'équipes de plus en plus polyvalents impliqués dans son traitement, qui comprennent des médecins, des infirmières, des psychologues, des nutritionnistes et d'autres professionnels de la santé (MARTINS; MIYAZAKI, 2019). Par conséquent, nous recherchons des alternatives qui rendent la prise en charge de la maladie plus appropriée.

La chirurgie bariatrique, dans ce contexte, est considérée par de nombreux patients comme le moyen le plus efficace de sortir de la lutte efficace contre l'obésité. Cependant, dans la période post-chirurgicale, il est nécessaire de maintenir le poids, car ses avantages incluent, en plus de l'ajustement de la composition corporelle, une diminution significative des comorbidités associées (FLORES, 2014). Les symptômes dépressifs et anxieux sont considérablement réduits, ainsi qu'une amélioration du fonctionnement sexuel, une augmentation du niveau d'activité et une amélioration intégrale de la qualité de vie liée à la santé (FLORES, 2014). RC: 98481 
Par rapport au contexte brésilien, le nombre de chirurgies liées à la perte de poids a progressivement augmenté, mais de manière expressive, ce qui implique la nécessité d'accorder une plus grande attention aux questions de santé et de bien-être social. Au détriment des critiques et controverses associées à la chirurgie bariatrique, il est recommandé que le patient remplisse certaines conditions préalables spécifiques avant de pouvoir y être soumis (GORDON; KAIO; SALLET, 2011). Selon les conditions du patient, il peut ou non devenir apte à la procédure. L'un des paramètres qui permettent de prendre cette décision est son suivi par l'équipe multidisciplinaire, ce qui implique également la présence de la famille et la volonté du patient de changer les habitudes et les comportements (FANDIÑO et al., 2004). Certaines comorbidités agissent comme des facteurs aggravants pour les non-susmentionnés bariatriques comme alternative à la résolution du problème.

Quelques exemples peuvent être cités, tels que la pneumopathie sévère, l'insuffisance rénale, les lésions myocardiques marquées et la cirrhose du foie (FANDIÑO et al., 2004). Cependant, le bariatrique est généralement bien accepté et recommandé. En ce qui concerne les recommandations pour l'intervention bariatrique, celles liées à la personne et aux comorbidités se démarquent. Au début des années 1990, le National Institute of Health des États-Unis a commencé à souligner l'importance de l'approche multidisciplinaire dans la période préopératoire, y compris, dans ce processus, l'évaluation psychologique avant l'opération, étant une partie essentielle et pour la prise de décision (FLORES, 2014). Le Consensus bariatrique brésilien et le Conseil fédéral de médecine ont également recommandé cette pratique, mais non sans avoir d'abord déterminé l'importance de la figure d'un psychologue et/ou d'un psychiatre dans l'équipe multidisciplinaire (COUTINHO, 1999).

Comme le rappelle le Conseil fédéral de la médecine, le professionnel responsable, a priori, du traitement de la santé mentale, compris ici comme le psychologue et/ou le psychiatre, fait partie d'une équipe qui assume une responsabilité fondamentale. II vise à fournir au patient une meilleure perception (critique) de son état de santé, ce qui contribue directement à un meilleur traitement adem (ROSA et al., 2020). Lors de l'évaluation multidisciplinaire préopératoire, en plus de faire une évaluation préalable 
de l'historique de santé personnelle et familiale, l'équipe doit se préoccuper, entre autres, de la pratique/utilisation ou non de substances psychoactives, de la présence de conditions psychotiques ou médicales, du niveau intellectuel et cognitif qui permet au patient d'être conscient des risques, les succès et les soins liés au processus chirurgical auquel il sera soumis, tels que les soins préopératoires, ainsi que l'attention devraient également être accordées aux aspects qui influencent la qualité de vie des patients et de leurs familles (FLORES, 2014).

Compte tenu de ce qui précède, il est nécessaire de discuter de la classification des chirurgies bariatriques, qui sont: restrictives ou disabsorptives.

Parmi les plus courantes, il existe une gastrectomie verticale, également appelée chirurgie Sleeve, étantune chirurgie restrictive qui enlève une grande partie de l'estomac (fond gastrique et corps), laissant un estomac résiduel en forme de mangue (tube linéaire) d'une capacité volumétrique de $150 \mathrm{ml}$. Les patients, avec cette procédure, subissent une réduction moyenne de $30 \%$ du poids total au cours des 2 premières années (COUTINHO, 1999).

La technique Capella, connue sous le nom de by-pass gastrique en $y$-Roux, consisteen une modalité qui rassemble la restriction à la désabsorption. II s'agit d'une gastroplastie associée à un shunt gastro-jéjunal sous la forme de la lettre $Y$ (Appel de $Y$ de Roux). La procédure réduit la taille de l'estomac afin qu'il soit capable de s'adapter à un volume inférieur à $30 \mathrm{ml}$ (MARTINS; MIYAZAKI, 2019). II fait également le lien avec une boucle intestinale plus distale (anastomose), conduisant à une zone disaborsotive étendue, car environ 3 mètres de mince sont détournés du transit alimentaire (FANDIÑO et al., 2004).

La technique de Scopinaro, à caractère plus désabsorbant, est une méthode qui consiste à appliquer un «by-pass » biliopancréatique partiel avec gastrectomie distale (FANDIÑO et al., 2004). Parce qu'elle a une capacité plus dénutritive et est plus désabsorbante, cette technique a été moins réalisée que les techniques Sleeve et Capella. 
L'objectif n'est pas de souligner la meilleure procédure, mais d'énumérer les possibilités existantes et de discuter des avantages et des moyens qui peuvent amener le patient à avoir une meilleure qualité de vie, car, entre autres choses, le principal objectif est de contribuer à la perte de poids, contribuant, en parallèle, à améliorer la santé des patients bariatriques.

\section{L'IMPORTANCE DU SUIVI NUTRITIONNEL ET MENTAL}

\subsection{SURVEILLANCE NUTRITIONNELLE}

La chirurgie bariatrique est une procédure qui ajoute des résultats positifs au traitement de l'obésité et des comorbidités associées. C'est un sujet qui a longtemps été discuté principalement par les médecins et les nutritionnistes, mais qui fait aujourd'hui partie d'une approche interdisciplinaire, comprenant la psychologie, la psychiatrie, l'endocrinologie, l'anesthésiologie, la cardiologie, la physiothérapie, les soins infirmiers et l'éducation physique (FAGUNDES; CAREGNATO; SILVEIRA, 2016). Étant donné que ces professionnels sont essentiels dans le traitement (TRAVADO et al., 2004).

Une étude de Silva et al. (2015) a cherché à identifier le profil des personnes qui ont choisi d'effectuer une chirurgie bariatrique comme alternative au traitement de l'obésité. Il a été conclu que, parmi les 100 personnes interrogées, 49 \% ont déclaré avoir opté pour la chirurgie en raison de l'échec des traitements antérieurs; 39\% au détriment des maladies associées; et, enfin, 12\% ont opté pour la procédure en raison de son efficacité et de sa facilité dans la perte de poids excédentaire (SILVA et al., 2015).

Les objectifs qui ont motivé les patients à se faire opérer en bariatrie indiquent que 87 $\%$ d'entre elles ont choisi de désirer une meilleure qualité de vie; 82 \% pour améliorer la santé; $34 \%$ pour les facteurs esthétiques; et $15 \%$ pour les facteurs sociaux (SILVA et al., 2015). Les patients qui ont subi six mois de suivi nutritionnel préopératoire ont eu une plus grande perte de poids que ceux qui ont arrêté de perdre du poids avant l'opération ou ont repris du poids perdu (KORTCHMAR et al., 2018). Compte tenu de RC: 98481 
ce scénario, on émet l'hypothèse qu'un suivi nutritionnel plus long pour les patients candidats à la chirurgie bariatrique peut les rétrograder, ce qui pourrait justifier les résultats trouvés par les deux études. En ce sens, les informations fournies par le professionnel nutritionniste peuvent promouvoir de nouvelles habitudes alimentaires, ce qui augmente les chances de succès de la chirurgie (ARAGÃO; ZAMBON, 2021).

La fréquence à laquelle ces patients ont des consultations avec des nutritionnistes est un facteur décisif pour le succès de la chirurgie. Ceux qui subissent ce suivi ont une plus grande perte de poids après la chirurgie (BARDAL; CECCATTO; MEZZOMO, 2016). Une récidive de poids a été observée chez $39 \%$ des patients soumis à une gastroplastie de réduction, en particulier à partir de 12 mois après l'intervention chirurgicale. En ce qui concerne la perte de poids préopératoire, $74 \%$ des patients ont présenté une perte de poids sans suivi nutritionnel, ce qui justifie la pertinence de ce professionnel dans la période postopératoire (BARDAL; CECCATTO; MEZZOMO, 2016). La réduction de poids dans la période préopératoire est bénéfique, car elle stimule l'adhésion du patient à des habitudes plus saines, car il sera plus conditionné à un régime alimentaire adéquat après la procédure. Cependant, d'autre part, la réduction de poids due à des méthodes non fondées, telles que la restriction alimentaire et le régime hypocalorique sans suivi nutritionnel, sont quelques-uns des aspects qui peuvent causer des situations de carences nutritionnelles courantes chez les patients obèses (RODRIGUES; FARIA, 2020). C'est le cas de la vitamine D et du profil de fer chez les femmes en âge de procréer (RODRIGUES et al., 2017).

Un autre facteur à prendre en compte dans l'évaluation est la réduction de la masse musculaire due à une restriction calorique sévère. Cela peut entraîner des troubles de la capacité fonctionnelle, une réduction de la synthèse des protéines et une diminution de la compétence immunitaire (BONAZZI et al., 2007). En outre, la réduction de poids préopératoire est basée sur la facilité transopératoire pour le chirurgien dans laquelle il y a une réduction de la graisse intra-abdominale et une réduction du volume du foie, qui, chez la plupart des patients qui souhaitent une intervention chirurgicale, est affectée par la satatose hépatique. D’autres études comprennent qu'une perte de poids supérieure à 9,5\% réduit significativement les complications par rapport à une 
perte de poids inférieure à $5 \%$ chez les patients ayant un IMC supérieur à $45 \mathrm{~kg} / \mathrm{m}^{2}$. Les patients ayant un IMC $>50 \mathrm{~kg} / \mathrm{m}^{2}$ peuvent bénéficier le plus en termes de technique chirurgicale (THIBAULT et al., 2016).

L'objectif du suivi nutritionnel avant la chirurgie est de sensibiliser à l'importance d'adhérer à de saines habitudes alimentaires, essentielles au maintien d'une perte de poids post-chirurgicale à long terme (GONÇALVES; KOHLSDORF; PEREZ-NEBRA, 2020). Compte tenu de ce scénario, les connaissances sur l'alimentation et la nutrition chez les personnes soumises et les candidats à la chirurgie bariatrique soulèvent des doutes fréquents, tels que la possibilité de vomissements, la satisfaction dans les 48 premières heures après la chirurgie, les changements possibles dans le goût et les conditions dépressives, ce qui renforce encore l'importance de la performance de l'équipe multidisciplinaire (NISHIYAMA et al., 2007). Une étude a révélé l'impact expressif du suivi interdisciplinaire sur l'efficacité de la chirurgie bariatrique et a démontré qu'il est d'une importance vitale de préparer l'individu avant d'effectuer la chirurgie (MASARI et al., 2012).

Le comportement alimentaire des personnes obèses est lié à l'environnement dans lequel vit l'individu. Des habitudes alimentaires inadéquates acquises tout au long de l'enfance corroborent l'augmentation de l'adiposité corporelle (NISHIYAMA et al., 2007). De plus, il y a une détérioration de la qualité de vie chez les personnes en surpoids, car il y a un plus grand risque de développer des comorbidités associées à l'obésité. Ainsi, les interventions nutritionnelles liées aux pratiques d'éducation nutritionnelle contribuent également à l'amélioration de la qualité de vie (BATISTA FILHO; RISSIN, 2003). Une étude qui a comparé le comportement alimentaire avec la préférence des individus dans la période pré et postopératoire de la chirurgie bariatrique a révélé que, parmi les patients en préparation préopératoire, 94\% avaient un « comportement de pincement ». Parmi les patients soumis à une intervention chirurgicale, $60 \%$ avaient ce même comportement (MACHADO et al., 2008).

Parmi les patients soumis à cette intervention chirurgicale, 33,3\% ont acquis de meilleures habitudes alimentaires après la chirurgie et $63 \%$ sont restés avec les mêmes habitudes identifiées avant la chirurgie, ce qui renforce encore l'importance du RC: 98481

Lien d'accès: https://www.nucleodoconhecimento.com.br/psychologie-fr/suivi-psychologique 
suivi nutritionnel (RODRIGUES et al., 2017). Avant la chirurgie, les patients avaient une préférence pour les aliments riches en calories, tels que les glucides simples (42\%), les bonbons (26\%) et les collations (14\%) (MACHADO et al., 2008). Cependant, après la chirurgie, l'étude a révélé que les patients recherchaient des aliments faciles à prendre, tels que des bonbons (45\%), des collations (22\%), des glucides (20\%) et une préférence accrue pour la bière (7\%) (MACHADO et al., 2008). La fréquence des épisodes de frénésie alimentaire chez les patients avant l'opération a augmenté en raison de l'augmentation de la nervosité, qui était un facteur de déséquilibre qui stimulait l'apport alimentaire excessif et $24 \%$ ont signalé la présence d'anxiété et / ou de troubles de l'humeur (dépression).

Les personnes obèses compulsives ont une plus grande prédisposition à devenir des «pincement " après une chirurgie bariatrique, car la préférence pour la consommation de bonbons et de collations est plus élevée chez les obèses compulsifs que chez les personnes obèses non compulsives (COLLES; DIXON; O'BRIEN, 2008). Les habitudes de «pincement » et la compulsion alimentaire elles-mêmes restent ou sont renforcées après la procédure, ce qui démontre en outre l'importance d'un suivi psychologique et / ou psychiatrique et nutritionnel dans les périodes pré- et postopératoires, car des habitudes inadéquates peuvent contribuer à la prise de poids chez ces patients. Ceux-ci peuvent être liés à la prévalence plus élevée de symptômes gastro-intestinaux, au syndrome de dumping, ainsi qu'à de graves dommages psychologiques (FLORES, 2014; MACHADO et al., 2008; GREENBERG; SOGG; PERNA, 2009). L'acte de manger est intrinsèquement lié à des stimuli internes et externes.

Les facteurs organiques, psychiques et sociaux sont pris en compte, et ainsi, on perçoit que l'apport alimentaire va au-delà des aspects nutritionnels, et réconcilie ainsi les motivations cachées liées aux conflits vécus et qui sont indépendantes de la sensation de faim (MACHADO et al., 2008; SOUTO; FERRO-BUCHER, 2006). La limitation causée par la chirurgie bariatrique peut entraîner des risques pour les individus compulsifs, ce qui peut entraver leur adaptation au nouvel état alimentaire, car la capacité gastrique sera plus faible et entraînera donc des dommages si l'individu 
continue à se nourrir de manière incorrecte (BOCCHIERI-RICCIARDI et al., 2006). Les personnes candidates à la chirurgie bariatrique comprennent la dynamique associée à la frénésie alimentaire. La recherche de nouvelles habitudes est un moyen de s'assurer que de nouveaux troubles ne prévaudront pas après la procédure. Les directives nutritionnelles doivent être clarifiées pour le patient.

Les sujets d'une extrême importance doivent être abordés afin que le succès postchirurgical soit remarqué, tels que la digestion et l'absorption des nutriments avant et après la procédure; lignes directrices sur la préparation de plats plus sains; la lecture des étiquettes des aliments industrialisés; les achats sur le marché; et, enfin, ces candidats devraient être instruits sur l'alimentation pendant les premiers mois après la chirurgie (BOCCHIERI-RICCIARDI et al., 2006). L'importance du nutritionniste pendant tout le suivi dans la période pré et postopératoire devient résumée. Afin d'obtenir des résultats positifs. L'évaluation préopératoire doit être strictement suivie, sur la base d'un protocole d'activité préalablement élaboré. II est nécessaire que le patient et l'équipe interdisciplinaire soient impliqués et conscients de l'importance de promouvoir des habitudes alimentaires plus saines (MASARI et al., 2012).

\subsection{SUIVI EN SANTÉ MENTALE}

Les aspects psychologiques peuvent influencer ou être une conséquence de l'obésité, ainsi que peuvent être responsables de la prise de poids ou des difficultés à maintenir le poids atteint après la chirurgie (VACCARO, 2018). Les cas d'incapacité à maintenir la perte de poids sont également associés au sentiment de manque de préparation aux changements nécessaires. Éviter l'échec du traitement bariatrique dépend d'une investigation pré-chirurgicale sur les antécédents alimentaires du patient, sur le rôle de la nourriture dans sa vie, ainsi que sur la nécessité d'enquêter et de prendre soin de l'impulsion qui conduit à la voracité de manger (EDLER, 2017). La chirurgie est capable de mincir le corps, cependant, certains problèmes psychologiques peuvent être négligés, et ils sont liés au contrôle de l'alimentation, à l'estime de soi et à l'adaptation à l'alimentation liée à l'émotion, ce qui implique de créer une identité avec le corps, un nouveau défi, car l'auto-évaluation, après perte de poids, est négative (FAGUNDES; CAREGNATO; SILVEIRA, 2016).

RC: 98481

Lien d'accès: https://www.nucleodoconhecimento.com.br/psychologie-fr/suivi-psychologique 
Cette construction négative est associée à l'image corporelle, à l'excès de peau, à la perception de soi dans le nouveau corps et à l'adaptation et à la reconstitution et à l'image de soi dans le corps maigre (ALEGRÍA; LARSEN, 2013). Le suivi et le soutien psychologique avant et après la chirurgie contribuent à l'amélioration des résultats des patients dans leur défi de perdre du poids, d'acquérir une meilleure qualité de vie et de réduire le désir de manger, étant ces facteurs cruciaux pour éviter la récurrence de l'obésité (HOLLYWOOD; OGDEN; PRING, 2012). Il y a aussi la réduction associée de la faim, la moindre préoccupation avec la nourriture, ainsi que le sentiment d'un plus grand contrôle concernant l'apport alimentaire.

Une étude a détecté que le type d'adaptation adopté par le patient après une chirurgie bariatrique détermine ou non le résultat de la perte de poids, et que les facteurs psychologiques et physiologiques se combinent et interagissent de manière significative, influençant ainsi les résultats (la construction d'une image plus positive sur le corps, par exemple) (FIGURA et al., 2015).

Les individus opérés peuvent passer par une période de restructuration émotionnelle, une phase dans laquelle il est essentiel de maintenir un suivi psychologique, à travers des thérapies, pour éviter les distorsions cognitives et les perceptions sur la réalité déformée. (MAGDALENO JR.; CHAIM; TURATO, 2008). Compte tenu de ce scénario, l'attention est attirée sur la nécessité d'un traitement psychologique individualisé dans la période pré- et post-chirurgicale. II est lié à l'identification et au traitement de la structure mentale de chaque patient, visant, avec ces soins, à éviter d'autres complications après la chirurgie (GORDON; KAIO; SALLET, 2011). Les expériences physiques, psychologiques, sociales et cliniques des patients subissant une chirurgie bariatrique influencent le processus de traitement. Parmi ceux qui touchent le plus ces sujets, on peut lire : les changements physiques et les défis subséquents, les expériences psychologiques, le soutien social et les expériences cliniques (LIU; IRWIN, 2017).

Il y a une amélioration des conditions psychologiques, physiques et sociales, cependant, il y a une préoccupation continue au sujet d'éventuels événements imprévus typiques des résultats de la chirurgie et l'importance de soutenir les services RC: 98481

Lien d'accès: https://www.nucleodoconhecimento.com.br/psychologie-fr/suivi-psychologique 
de santé recommandés pour la période post-chirurgicale, y compris, dans ce processus, le soutien psychologique, puisque les patients doivent apprendre à faire face aux changements dans le corps, les relations sociales et la construction d'une nouvelle identité (LIU; IRWIN, 2017). Les proches ou les soignants du patient bariatrique ont également besoin d'un soutien psychologique (SPRENGEL, 2015), car le soutien social à toutes les personnes impliquées implique un meilleur respect des comportements de santé plus sains, tels que l'activité physique, une alimentation plus saine, la réduction des situations de stress (MARTINS; MIYAZAKI, 2019). Ainsi, l'amélioration de la santé est encouragée, y compris la santé mentale et la qualité de vie (ABREU RODRIGUES; SEIDL, 2015).

Cependant, l'efficacité du traitement nécessite certains soins, tels que le temps de préparation psychologique avant et après la chirurgie, tant pour le patient que pour les conjoints et les membres de la famille, car ce sont eux qui les accompagneront tout au long du traitement pré- et post-bariatrique (DELAPRIA, 2019). La motivation et le soutien à long terme des patients doivent être stimulés dans le processus de suivi psychologique (MARCHESINI, 2010). Dans ce voyage, il est d'une importance primordiale d'inclure la famille et les amis dans le processus préopératoire, ainsi que d'assurer la clarté du patient concernant les gains et les pertes impliqués dans la procédure (VACCARO, 2018). Le traitement nécessite donc la performance d'une équipe multidisciplinaire. Celui-ci doit comprendre la subjectivité de chaque cas, en prévoyant et en accompagnant les facteurs qui, directement ou indirectement, peuvent influencer le succès ou l'échec du processus, ce qui implique de redimensionner certaines stratégies pour atteindre le succès (MARCELINO; PATRÍCIO, 2011).

La chirurgie bariatrique, en fait, offre une meilleure qualité de vie au patient, ainsi que favorise l'amélioration de la socialisation due à la perte de poids, cependant, il convient de souligner que, dans ce processus de traitement, le patient devra toujours faire face à des problèmes psychiques et nutritionnels afin d'atteindre la qualité de vie souhaitée (DELAPRIA, 2019). Le soutien est essentiel pour que les changements de comportement alimentaire et de mode de vie puissent être rendus possibles, ainsi que les changements corporels, sociaux et identitaires (VACCARO, 2018). Le maintien et 
la continuité du poids grâce au traitement chirurgical dépendent de l'action, de la conscience et de l'engagement du patient, ce qui peut être difficile et irréalisable sans suivi psychologique et nutritionnel.

Le patient lui-même doit se rendre compte qu'il est l'agent de transformation. La chirurgie amincit, mais le corps maigre dépend de changements internes, une demande qui va au-delà de l'estomac. L'adaptation aux nouvelles dimensions du corps et l'ajustement des relations sociales, ainsi que la construction d'une nouvelle identité et la déconnexion de l'impulsion à manger sont des questions qui peuvent survenir après la chirurgie, et nécessitent donc plus d'attention et de soins (GORDON; KAIO; SALLET, 2011). La psychothérapie peut contribuer à l'esprit en suivant le processus d'amincissement du corps.

\section{CONSIDÉRATIONS FINALES}

Cette étude visait à démontrer l'importance du suivi nutritionnel et mental avant et après la chirurgie bariatrique. Les preuves indiquent que le rôle joué par le psychologue dans l'équipe multidisciplinaire, en particulier dans la période postopératoire des patients bariatriques, en ce qui concerne le contrôle et / ou la réduction de l'anxiété et des symptômes dépressifs, et d'autres changements psychopathologiques qui nuisent à la santé mentale, est essentiel, ce qui rend le débat sur le rôle des psychologues et autres professionnels impliqués fondamental. La pertinence de ces professionnels est liée au fait qu'une action multidisciplinaire permettra au patient soumis au bariatre de s'habituer à un nouveau niveau de vie (habitudes alimentaires, relation avec la nourriture et le corps) et, dans ce processus, un suivi psychologique pendant la période pré et postopératoire est pertinent pour éviter des problèmes tels que la prise de poids.

L'évaluation psychologique des patients bariatriques est pertinente, car, de cette façon, il est possible de comprendre ce patient de manière holistique et, par conséquent, une réduction des complications mentales de la chirurgie pouvant nuire à la qualité de vie du patient est favorisée. La nécessité d'une évaluation psychologique approfondie devient un résumé. Le patient doit être étudié et éduqué afin que les RC: 98481

Lien d'accès: https://www.nucleodoconhecimento.com.br/psychologie-fr/suivi-psychologique 
changements successifs à la chirurgie soient efficaces et visualisés à long terme. Les indications et contre-indications liées à la chirurgie bariatrique doivent respecter l'évaluation de l'équipe multidisciplinaire, étant donné que, en raison des collections et des pressions quotidiennes, les problèmes affectant la santé mentale, tels que les troubles anxieux, tels que les troubles anxieux, parmi lesquels se distinguent la phobie sociale, l'anxiété généralisée et le trouble panique, en ce qui concerne les troubles de I'humeur, le nombre croissant de personnes touchées par la dépression est inquiétant.

De tels troubles sont devenus fréquents et affectent la vie de nombreuses personnes. C'est au milieu de ce processus, d'une certaine manière, turbulent, que se trouve le patient post-bariatrique, qui doit faire face, presque toujours, à un fardeau émotionnel au-delà du supportable, qui finit par créer des troubles inattendus associés à l'anxiété. En ce sens, le psychologue joue un rôle très important depuis le début du processus chirurgical, mais sa performance ne se limite pas à lui. Par conséquent, sa contribution dans la période postopératoire est essentielle.

\section{RÉFÉRENCES}

ABREU-RODRIGUES, M.; SEIDL, E. M. F. Apoio social e reganho de peso póscirurgia bariátrica: estudo de caso sobre intervenção com cuidador. Temas em Psicologia, v. 23, n. 4, p. 1003-1016, 2015.

\section{AILLS, L. et al. ASMBS allied health nutritional guidelines for the surgical weight} loss patient: surgery for obesity and related diseases. Surg Obes Relat Dis., v. 4, n. 5, p. 73-108, 2008.

ALEGRÍA, C. A.; LARSEN, B. "That's who I am: A fat person in a thin body": Weight loss, negative self-evaluation, and mitigating strategies following weight loss surgery. Journal of the American Association of Nurse Practitioners, v. 27, n. 3, p. 137144,2013

ARAGÃO, C. N.; ZAMBON, E. A avaliação psicológica para cirurgia bariátrica - uma análise teórica e prática. Rumos da inFormação, v. 2, n. 1, p. 63-76, 2021. 
BARDAL, A. G.; CECCATTO, V.; MEZZOMO, T. R. Fatores de risco para recidiva de peso no pós-operatório tardio de cirurgia bariátrica. Scientia médica, v. 26, n. 4, p. ID24224-ID24224, 2016.

BATISTA FILHO, M.; RISSIN, A. A transição nutricional no Brasil: tendências regionais e temporais. Cadernos de saúde pública, v. 19, p. S181-S191, 2003.

BOCCHIERI-RICCIARDI, L. E. et al. Pre-surgery binge eating status: effect on eating behavior and weight outcome after gastric bypass. Obesity surgery, v. 16, n. 9, p. 1198-1204, 2006.

BONAZZI, C. L. et al. A intervençâo nutricional no pré e pós operatório da cirurgia bariátrica. RBONE-Revista Brasileira de Obesidade, Nutrição e Emagrecimento, v. 1, n. 5, p. 59-69, 2007.

BRASIL. Ministério da Saúde. Vigitel 2016: vigilância de fatores de risco e proteção para doenças crônicas por inquérito telefônico. Brasília: Ministério da Saúde, 2017.

BUCHWALD, $\mathrm{H}$. et al. Bariatric surgery: a systematic review and meta-analysis. Jama, v. 292 , n. 14 , p. 1724-1737, 2004.

CAMPOS, J. et al. O papel da cirurgia metabólica para tratamento de pacientes com obesidade grau ie diabete tipo 2 não controlados clinicamente. ABCD: Arquivos Brasileiros de Cirurgia Digestiva (São Paulo), v. 29, p. 102-106, 2016.

COLLES, S. L.; DIXON, J. B.; O'BRIEN, P. E. Loss of control is central to psychological disturbance associated with binge eating disorder. Obesity, v. 16, n. 3, p. 608-614, 2008.

COOPER, P. L. et al. Nutritional consequences of modified vertical gastroplasty in obese subjects. International journal of obesity, v. 23, n. 4, p. 382-388, 1999.

COSTA, A. C. C. et al. Obesidade em pacientes candidatos a cirurgia bariátrica. Acta Paulista de enfermagem, v. 22, p. 55-59, 2009. 
COSTA, D. Eficiência do acompanhamento nutricional no pré e pós-operatório da cirurgia bariátrica. RBONE-Revista Brasileira de Obesidade, Nutrição e Emagrecimento, v. 7, n. 39, 2013.

COUTINHO, W. Consenso latino-americano de obesidade. Arquivos Brasileiros de Endocrinologia \& Metabologia, v. 43, n. 1, p. 21-67, 1999.

DELAPRIA, A. M. T. A importância do acompanhamento psicológico no pré e pósoperatório da cirurgia bariátrica. Revista UNINGÁ, v. 56, n. S1, p. 78-88, 2019.

DOLNE, F.; SOUZA, J. S. M. de.; MATTE, J. Atuação da enfermagem na assistência prestada ao paciente obeso mórbido no pós-operatório de cirurgia bariátrica. RBONERevista Brasileira De Obesidade, Nutrição E Emagrecimento, v. 14, n. 87, p. 550$559,2020$.

EDHOLM, D. et al. Preoperative 4-week low-calorie diet reduces liver volume and intrahepatic fat and facilitates laparoscopic gastric bypass in morbidly obese. Obesity surgery, v. 21, n. 3, p. 345-350, 2011.

EDLER, S. Tempos Compulsivos: a busca desenfreada pelo prazer. Rio de Janeiro: Casa da Palavra, 2017. 176 pgns.

FAGUNDES, M. A. B. G.; CAREGNATO, R. C. A.; SILVEIRA, L. M. de. O. B. Variáveis psicológicas associadas à cirurgia bariátrica. Aletheia, v. 49, n. 2, p. 47-54, 2016.

FANDIÑO, J. et al. Cirurgia bariátrica: aspectos clínico-cirúrgicos e psiquiátricos. Revista de psiquiatria do Rio Grande do Sul, v. 26, n. 1, p. 47-51, 2004.

FIGURA, A. et al. Determinants of weight loss following laparoscopic sleeve gastrectomy: the role of psychological burden, coping style, and motivation to undergo surgery. Journal of obesity, v. 2015, p. 1-10, 2015.

FLORES, C. A. Avaliação psicológica para cirurgia bariátrica: práticas atuais. ABCD: Arquivos Brasileiros de Cirurgia Digestiva (São Paulo), v. 27, p. 59-62, 2014. 
GONÇALVES, S. J. B. G.; KOHLSDORF, M.; PEREZ-NEBRA, A. R. Adesão ao pósoperatório em cirurgia bariátrica: análise sistemática da literatura brasileira. Psicologia Argumento, v. 38, n. 102, p. 626-646, 2020.

GORDON, P. C.; KAIO, G. H.; SALLET, P. C. Aspectos do acompanhamento psiquiátrico de pacientes obesos sob tratamento bariátrico: revisão. Archives of Clinical Psychiatry (São Paulo), v. 38, p. 148-154, 2011.

GREENBERG, I.; SOGG, S.; PERNA, F. M. Behavioral and psychological care in weight loss surgery: best practice update. Obesity, v. 17, n. 5, p. 880-884, 2009.

HOLLYWOOD, A.; OGDEN, J.; PRING, C. The impact of a bariatric rehabilitation service on weight loss and psychological adjustment-study protocol. BMC Public Health, v. 12, n. 1, p. 1-5, 2012.

KAC, G.; SICHIERI, R.; GIGANTE, D. P. Epidemiologia nutricional. São Paulo: Editora Fiocruz, 2007. 580 pgns.

$\mathrm{KIM}$, J. et al. ASMBS updated position statement on insurance mandated preoperative weight loss requirements. Surgery for Obesity and Related Diseases, v. 12, n. 5, p. 955-959, 2016.

KORTCHMAR, E. et al. Reganho de peso após a cirurgia bariátrica: um enfoque da fenomenologia social. Acta Paulista de Enfermagem, v. 31, n. 4, p. 417-422, 2018.

KULICK, D.; HARK, L.; DEEN, D. The bariatric surgery patient: a growing role for registered dietitians. Journal of the American Dietetic Association, v. 110, n. 4, p. 593-599, 2010.

LIU, R. H.; IRWIN, J. D. Understanding the post-surgical bariatric experiences of patients two or more years after surgery. Quality of Life Research, v. 26, n. 11, p. 3157-3168, 2017.

MACHADO, C. E. et al. Compulsão alimentar antes e após a cirurgia bariátrica. ABCD: Arquivos Brasileiros de Cirurgia Digestiva (São Paulo), v. 21, p. 185-191, 2008. RC: 98481 
MAGDALENO JR, R.; CHAIM, E. A.; TURATO, E. R. Características psicológicas de pacientes submetidos à cirurgia bariátrica. Revista de Psiquiatria do Rio Grande do Sul, v. 31, p. 73-78, 2009.

MAGDALENO JR, R.; CHAIM, E. A.; TURATO, E. R. Psychological characteristics of patients submitted to bariatric surgery. Revista de Psiquiatria do Rio Grande do Sul, v. 31, p. 73-78, 2009.

MARCELINO, L. F.; PATRICIO, Z. M. A complexidade da obesidade e o processo de viver após a cirurgia bariátrica: uma questão de saúde coletiva. Revista Ciência e Saúde Coletiva, v. 16, n. 12, p. 4767-4776, 2011.

MARCHESINI, S. D. Late psychological follow-up on patients submitted to bariatric surgery. ABCD: Arquivos Brasileiros de Cirurgia Digestiva, v. 23, n. 2, p. 108-113, 2010.

MARTINS, C. E. P.; MIYAZAKI, E. T. A presença do psicólogo na equipe multidisciplinar e a ansiedade pós-cirurgia bariátrica. Revista Campo do Saber, v. 5, n. 2, p. 77-90, 2019.

MASARI, S. P. et al. Preparação multidisciplinar pré-cirúrgia bariátrica na visão do cliente. RBONE-Revista Brasileira de Obesidade, Nutrição e Emagrecimento, v. 6, n. 32, p. 1-13, 2012.

MELO, M. E. Os Números da Obesidade no Brasil: VIGITEL 2009 e POF 20082009. 2011. Disponível em: https://www.saudedireta.com.br/docsupload/13403687290besidade\%20no\%20Brasil \%20VIGITEL\%202009\%20POF2008_09\%20\%20II.pdf. Acesso em: 02 ago. 2021.

MENEGOTTO, A. L. S. et al. Avaliação da frequência em consultas nutricionais dos pacientes após cirurgia bariátrica. ABCD: Arquivos Brasileiros de Cirurgia Digestiva (São Paulo), v. 26, p. 117-119, 2013. 
MENSORIO, M. S. Análise de estratégias de enfrentamento, ansiedade e hábitos em pacientes elegíveis à cirurgia bariátrica, com e sem acompanhamento psicológico. 2013. 159f. Dissertação (Mestrado em Processos de Desenvolvimento Humano e Saúde) - Universidade de Brasília, Brasília, DF, 2013.

NISHIYAMA, M. F. et al. Avaliação do nível de conhecimento e aderência da conduta nutricional em pacientes submetidos e candidatos à cirurgia bariátrica. Arquivos de Ciências da Saúde da UNIPAR, v. 11, n. 2, p. 89-98, 2007.

RASOULI, N. et al. Ectopic fat accumulation and metabolic syndrome. Diabetes, Obesity and Metabolism, v. 9, n. 1, p. 1-10, 2007.

ROCHA, C.; COSTA, E. Aspectos psicológicos na obesidade mórbida: Avaliação dos níveis de ansiedade, depressão e do auto-conceito em obesos que vão ser submetidos à cirurgia bariátrica. Analise psicológica, v. 30, n. 4, p. 451-466, 2012.

RODRIGUES, G. et al. Acompanhamento nutricional no pré-operatório de cirurgia bariátrica: tempo de seguimento versus redução de peso. Revista PsicoFAE: Pluralidades em Saúde Mental, v. 6, n. 2, p. 97-112, 2017.

RODRIGUES, J. O.; FARIA, H. M. C. Os aspectos psicossociais da cirurgia bariátrica: do pré ao pós-operatório. Cadernos de Psicologia, v. 2, n. 4, p. 551-569, 2020.

ROSA, T. M. F. et al. Atendimento nutricional em grupo de pacientes candidatos a cirurgia bariátrica: repercussões no consumo alimentar. RBONE-Revista Brasileira De Obesidade, Nutrição E Emagrecimento, v. 14, n. 87, p. 690-698, 2020.

SILVA, M. A da. Emagrecimento induzido por cirurgia: ação do psicólogo após a cirurgia bariátrica. Bol. Psicol., v. 65, n. 143, p. 243-246, 2015.

SILVA, P. T. da. et al. Perfil de pacientes que buscam a cirurgia bariátrica. ABCD: Arquivos Brasileiros de Cirurgia Digestiva (São Paulo), v. 28, p. 270-273, 2015.

SOCIEDADE BRASILEIRA DE CIRURGIA BARIÁTRICA E METABÓLICA (SBCBM). 2017. Disponível em: http://www.sbcbm.org.br/. Acesso em: 02 ago. 2021. RC: 98481 
SOUTO, S.; FERRO-BUCHER, J. S. N. Práticas indiscriminadas de dietas de emagrecimento e o desenvolvimento de transtornos alimentares. Revista de Nutrição, v. 19, p. 693-704, 2006.

SPRENGEL, A. L. Cirurgia Bariátrica: Manual de instruções para pacientes e familiares. São Paulo: M. Books do Brasil, 2015. 96 pgns.

THIBAULT, R. et al. Twelve key nutritional issues in bariatric surgery. Clinical nutrition, v. 35, n. 1, p. 12-17, 2016.

TRAVADO, L. et al. Abordagem psicológica da obesidade mórbida: Caracterização e apresentação do protocolo de avaliação psicológica. Análise psicológica, v. 22, n. 3, p. 533-550, 2004.

VACCARO, R. V. Acompanhamento psicológico e manutenção da perda de peso após a cirurgia bariátrica do tipo Bypass. 2018. 81f. Dissertação (Mestrado em Psicologia Clínica) - Pontifícia Universidade Católica de São Paulo, São Paulo, SP, 2018.

Publié: Octobre 2021.

Approuvé: Octobre 2021. 